\title{
Prohexadione-calcium Inhibits Apple, but not Peach, Tree Growth, but Has Little Influence on Apple Fruit Thinning or Quality
}

\author{
Ross E. Byers ${ }^{1}$ and K.S. Yoder ${ }^{2}$ \\ Alson H. Smith, Jr. Agricultural Research and Extension Center, Virginia Polytechnic Institute and State \\ University, 595 Laurel Grove Road, Winchester, VA 22602
}

In northern Virginia, over $80 \%$ of the apple crop is grown for processing. Many trees are propagated on vigorous rootstocks and require much pruning, especially in the tops. To reduce costs and labor needs, many growers prune every second or third year. When trees are not pruned, shading caused by growth in the current season or in nonpruned years is detrimental to pest control, fruit quality, and yield.

Several plant growth regulators have been evaluated for their potential to reduce vegetative growth of tree fruits, thereby reducing pruning costs and improving fruit quality. Several reviews (Faust, 1984; Looney, 1983; Luckwill, 1970; Miller, 1988; Williams, 1984) dealing with many of these compounds indicate that most have several effects of greater commercial value than control of vegetative growth.

The objectives of the experiments reported here were to evaluate the effects of prohexadione-calcium (3-oxido-4-propionyl-5-oxo-3cyclohexene-carboxylate), formulated as BAS-125 ("Apogee"®), on a) vegetative growth of apple and peach trees, b) fruit size and quality, and c) response to thinning chemicals.

\section{MATERIALS AND METHODS}

General. Randomized complete-block designs were used in all experiments. Trees were blocked by location within rows, by rootstock, and/or by flower density rating. All experiments had six blocks except for Expts. 1, 4, 5, and 6. Trees were sprayed with either a low pressure hand-wand sprayer or a Swanson three-point-hitch airblast sprayer with both fans adjusted to one side, which doubled air output and increased nozzle numbers.

In several experiments (Expts. 1-4), a 10-fruit sample was collected from each tree at harvest for quality evaluations, which included flesh firmness, soluble solids concentration, starch staining, percentage of red color, and incidence of water core. Flesh firmness of both peach and apple fruit was measured on two sides of each fruit with an Effegi penetrometer (model FT327; McCormick Fruit Tech, Yakima, Wash.) fitted with an 11.1-mm tip. Soluble solids concentration (SSC) was estimated with an Atago hand-held refractometer (model N1; McCormick Fruit Tech), utilizing a composite sample of juice resulting from penetrometer testing for each replicate of each treatment. Each apple fruit was cut in half transversely, and severity of water core was rated on a scale of 0 to $5(0=$ none, $5=$ severe $)$. Flesh starch was evaluated by dipping half of each apple in a solution of $\mathrm{I}_{2} / \mathrm{KI}$ for $\approx 15$ $\mathrm{s}$. The degree of staining was rated on a scale of 0 to 5 where $1=$ staining of the entire cut surface and $5=$ absence of starch (Poapst et al., 1959).

In the dormant season (Expts. 1-4), average shoot length of the longest top two shoots, length of the five longest scaffold shoots, total length of shoots longer than $30 \mathrm{~cm}$, weight and basal and terminal shoot diameters of these scaffold shoots, nodes $/ \mathrm{cm}$ of the basal $40 \mathrm{~cm}$, nodes $/ \mathrm{cm}$ of the upper $30 \mathrm{~cm}$ of shoots, and time required to prune each tree, number of cuts/tree, and pruning weights $/ \mathrm{cm}^{2}$ trunk cross-sectional area (TCSA) per tree were recorded.

Prohexadione-calcium was formulated as BAS 125 10W in 199597. Regulaid ${ }^{\circledR}$ (a surfactant mixture, polyoxyethylenepolypropoxy-

Received for publication 14 July 1998. Accepted for publication 11 Aug. 1998. This project was partially supported by BASF Corp., P.O. Box 13528, Research Triangle Park, NC 27709. The cost of publishing this paper was defrayed in part by the payment of page charges. Under postal regulations, this paper therefore must be hereby marked advertisement solely to indicate this fact.

${ }^{1}$ Professor of Horticulture.

${ }^{2}$ Associate Professor of Plant Pathology propanol, alkyl 2-ethoxethanol, and dihydroxy propane) was used with all prohexadione-calcium treatments either at $0.125 \%(\mathrm{v} / \mathrm{v})$ or as indicated.

Data were analyzed by ANOVA and GLM procedures using SAS software (SAS Institute, 1985). Means were compared by singledegree-of-freedom contrasts, or by linear and polynomial regressions, depending upon the experimental design.

Expt. 1. In 1994, sixteen 13-year-old 'Stayman'/seedling trees (four blocks) were used for four treatments. Four concentrations of prohexadione-calcium were applied $\left(0,125,250\right.$, or $\left.375 \mathrm{mg} \cdot \mathrm{L}^{-1}\right)$ at $2805 \mathrm{~L} \cdot \mathrm{ha}^{-1} 23 \mathrm{~d}$ after full bloom (AFB) (Table 1). In addition to fruit quality and dormant season shoot measurements previously described, five vegetative shoots selected at petal fall were measured on the periphery of each tree at intervals from 2 May to $7 \mathrm{Dec}$. For determining fruit cracking, three limbs having a total of $\approx 30$ fruit per tree were examined at weekly intervals and cracked fruit were removed from 25 July to 13 Oct. On 13 Oct., the remaining noncracked fruit were counted and the percentage of fruit cracked was determined.

Expt. 2. In 1995, thirty-six 10-year-old 'Starkrimson'/MM.106 or 'Starkrimson'/MM.111 trees (6 blocks) were used for six treatments. Prohexadione-calcium was applied at $0,125,250$, or $500 \mathrm{mg} \cdot \mathrm{L}^{-1}$ at $1870 \mathrm{~L} \cdot \mathrm{ha}^{-1}$, at $250 \mathrm{mg} \cdot \mathrm{L}^{-1}$ at $935 \mathrm{~L} \cdot \mathrm{ha}^{-1}$, at $500 \mathrm{mg} \cdot \mathrm{L}^{-1}$ at $468 \mathrm{~L} \cdot \mathrm{ha}^{-1} 23$ d AFB (Table 2). Fruit quality and dormant season shoot measurements were taken as previously described for evaluation of fruit characteristics. In addition, a 1-bushel $(20 \mathrm{~kg})$ sample was also passed over a Durand Wayland Omni Sort weight-sizer grader (Durand Wayland, LaGrange, Ga.) and the percentages of fruit in each weight category were determined.

Expt. 3. In 1996, thirty-six 10-year-old 'Starkrimson'/MM.106 or 'Starkrimson'/MM. 111 trees (three blocks MM.106 and three blocks MM.111 for a total of six blocks) were used for six treatments. Prohexadione-calcium at $250 \mathrm{mg} \cdot \mathrm{L}^{-1}\left(1870 \mathrm{~L} \cdot \mathrm{ha}^{-1}\right)$ was applied on 1) 6 May; 2) 17 May; 3 ) 17 May + June 3; 4) 17 May, 3 June, and 3 July; or 5) 17 May, 3 June, 3 July, and 24 July (Table 3). Additional trees served as controls. Fruit quality and dormant season shoot measurements were taken as previously described. A 10-fruit sample was collected 1 Oct. and $15 \mathrm{Nov}$. from each tree for evaluation of fruit characteristics.

Expt. 4. Forty 10-year-old 'Ace Delicious'/MM.111 trees (eight blocks) were used for five treatments. Prohexadione-calcium treatments (Table 4) were applied with an airblast sprayer at $1870 \mathrm{~L} \cdot \mathrm{ha}^{-1}$. Fruit quality and dormant season shoot measurements were taken as previously described. A 10-fruit sample was collected 1 Oct. from each tree for evaluation of fruit characteristics.

Expt. 5. In 1995, seventy 5-year-old 'Fuji'/M. 27 trees (seven blocks) were used for 10 treatments. Spray thinning chemical combinations (Table 5), included 1-naphthalenyl methylcarbamate (carbaryl), 6-benzyladenine + gibberellins $\mathrm{A}_{4+7}\left(\right.$ Accel $\left.^{\circledR}\right)$, 2chloroethylphosphonic acid (ethephon), oil (Sun 6E, 70 second superior spray oil typically used as a delayed dormate oil), methyl $N^{\prime} N^{\prime}-$ dimethyl- $N\left[\right.$ (methyl carbamol)oxy]-1-thiooxamimidate (Oxamyl ${ }^{\circledR}$ ), and trisiloxane ethoxylate methyl ether (a silicon surfactant, Silwet L77) were applied with or without prohexadione-calcium with a lowpressure hand wand sprayer on 15 May when fruit were $\approx 10 \mathrm{~mm}$ in diameter. The number of fruit on each tree was counted $15 \mathrm{~d}$ after treatment and expressed as number of fruit per $\mathrm{cm}^{2}$ trunk crosssectional area (TCSA). Ten fruit were harvested from each tree on 23 Oct. The fruit length $(\mathrm{L})$ and diameter (D) were determined by aligning 
Table 1. Effects of prohexadione-calcium on 'Stayman' apple tree growth in $1994 .^{2}$

\begin{tabular}{|c|c|c|c|c|c|c|c|c|c|c|}
\hline \multirow{2}{*}{$\begin{array}{l}\text { Prohexadione- } \\
\text { calcium } \\
\left(\mathrm{mg} \cdot \mathrm{L}^{-1}\right)\end{array}$} & \multicolumn{4}{|c|}{$\begin{array}{l}\text { Change in shoot length }(\mathrm{cm}) \\
\text { from } 2 \text { May until: }\end{array}$} & \multirow{2}{*}{$\begin{array}{c}\text { Fruit/cm }{ }^{2} \\
\text { limb } \\
\mathrm{CSA}^{\mathrm{x}} \\
25 \mathrm{July}\end{array}$} & \multicolumn{2}{|c|}{ Avg shoot ${ }^{y}$} & \multirow{2}{*}{$\begin{array}{c}\text { Avg } \\
\text { internode } \\
\text { length } \\
(\mathrm{cm})\end{array}$} & \multicolumn{2}{|c|}{ Shoot base ${ }^{y}$} \\
\hline & 30 June & 29 July & 31 Aug. & $7 \mathrm{Dec}$. & & $\begin{array}{l}\text { Length } \\
(\mathrm{cm})\end{array}$ & $\begin{array}{l}\mathrm{Wt} \\
(\mathrm{g})\end{array}$ & & $\begin{array}{l}\text { Diam } \\
(\mathrm{mm})\end{array}$ & $\begin{array}{l}\mathrm{CSA} \\
\left(\mathrm{mm}^{2}\right)\end{array}$ \\
\hline 125 & 13.5 & 21.3 & 29.4 & 28.5 & 3.48 & 99.6 & 48.8 & 2.4 & 9.1 & 6.60 \\
\hline 250 & 14.0 & 18.9 & 25.0 & 26.3 & 4.14 & 94.0 & 40.4 & 2.3 & 8.7 & 6.07 \\
\hline 375 & 14.2 & 17.2 & 22.8 & 23.8 & 4.00 & 92.2 & 41.9 & 2.3 & 9.2 & 6.73 \\
\hline \multicolumn{11}{|l|}{ Regression } \\
\hline
\end{tabular}

${ }^{2}$ Full bloom occurred 22 Apr. 1994. Treatments were applied 2 May when fruit were $7.4 \pm 0.60 \mathrm{~mm}$ in diameter. Airblast sprayer was calibrated for $2805 \mathrm{~L} \cdot \mathrm{ha} \mathrm{C}^{-1}$. Tree spacing was $7.9 \mathrm{~m}$ between rows, $5.2 \mathrm{~m}$ tree width, and $4.9 \mathrm{~m}$ tree height, equivalent to $80 \%$ tree row volume (TRV).

y Mean for longest 10 shoots in top of tree, measured 7 Dec.

${ }^{\mathrm{x}} \mathrm{CSA}=$ cross-sectional area.

Ns, ********Nonsignificant or significant at $P \leq 0.05,0.01$, or 0.001 , respectively.

Table 2. Effects of prohexadione-calcium applied in 1995 on dormant season measurements and pruning of 'Starkrimson'/MM.106 and 'Starkrimson'/MM.111 trees in Jan. 1996. ${ }^{2}$

\begin{tabular}{|c|c|c|c|c|c|c|c|c|c|c|c|c|c|}
\hline \multirow[b]{4}{*}{ Treatment } & \multirow{4}{*}{$\begin{array}{c}\text { Prohexadione- } \\
\text { calcium } \\
\left(\mathrm{mg} \cdot \mathrm{L}^{-1}\right)\end{array}$} & \multirow{4}{*}{$\begin{array}{l}\text { Water } \\
\text { applied } \\
\left(\mathrm{L} \cdot \mathrm{ha}^{-1}\right)\end{array}$} & & & & & & \multirow{4}{*}{$\begin{array}{l}\text { Basal } \\
\text { diam } \\
(\mathrm{cm})\end{array}$} & \multirow{2}{*}{\multicolumn{2}{|c|}{ Nodes/m }} & \multicolumn{3}{|c|}{ Pruning } \\
\hline & & & \multirow{2}{*}{\multicolumn{3}{|c|}{ Shoot length $(\mathrm{cm})$}} & \multirow{2}{*}{\multicolumn{2}{|c|}{ Shoot wt (g) }} & & & & \multirow{3}{*}{$\begin{array}{c}\text { Time } \\
\left(\mathrm{min} / \mathrm{cm}^{2}\right. \\
\left.\mathrm{TCSA}^{\mathrm{v}}\right)\end{array}$} & \multirow{3}{*}{$\begin{array}{l}\mathrm{Wt}\left(\mathrm{g} \cdot \mathrm{cm}^{-2}\right. \\
\text { TCSA })\end{array}$} & \multirow{3}{*}{$\begin{array}{l}\text { No cuts/ } \\
\mathrm{cm}^{2} \text { TCSA }\end{array}$} \\
\hline & & & & & & & & & \multirow{2}{*}{$\begin{array}{c}\text { Basal } 40 \\
\mathrm{~cm}\end{array}$} & \multirow{2}{*}{$\begin{array}{c}\text { Upper } 30 \\
\mathrm{~cm}\end{array}$} & & & \\
\hline & & & Top ${ }^{y}$ & Scaffold $^{x}$ & $>30 \mathrm{~cm}^{\mathrm{w}}$ & Top $^{y}$ & scaffold $^{x}$ & & & & & & \\
\hline 2 & 125 & 1870 & 134 & 109 & 386 & 193 & 294 & 1.29 & 68 & 52 & 2.88 & 73 & 1.05 \\
\hline 3 & 250 & 1870 & 123 & 103 & 360 & 165 & 259 & 1.24 & 74 & 52 & 3.12 & 84 & 1.28 \\
\hline 4 & 500 & 1870 & 118 & 95 & 367 & 147 & 182 & 1.13 & 85 & 50 & 2.88 & 63 & 1.26 \\
\hline \multicolumn{14}{|c|}{ Comparisons: } \\
\hline 0 vs. 125 & & & NS & NS & NS & NS & NS & $*$ & $* * *$ & $* * *$ & $*$ & $* *$ & $* *$ \\
\hline 0 vs. 250 & & & NS & $* *$ & NS & NS & $* * *$ & $* * *$ & $* * *$ & $* * *$ & NS & NS & NS \\
\hline 0 vs. 500 & & & NS & $* *$ & $*$ & * & $* * *$ & $* * *$ & $* * *$ & $* * *$ & $*$ & $* *$ & NS \\
\hline 125 vs. 500 & & & NS & $* *$ & NS & NS & ** & $* *$ & $* * *$ & NS & NS & NS & NS \\
\hline \multicolumn{14}{|c|}{125 (1870 water rate) vs. } \\
\hline \multicolumn{14}{|c|}{$\begin{array}{l}\text { Chemical rate regression: } \\
(0,125,250,500)\end{array}$} \\
\hline Q & & & NS & $* * *$ & NS & NS & $* * *$ & $* * *$ & $* * *$ & $* * *$ & NS & NS & NS \\
\hline \multicolumn{14}{|c|}{$\begin{array}{c}\text { Water rate regression } \\
(\text { treatments } 6,5,2)\end{array}$} \\
\hline $\mathrm{L}$ & & & NS & NS & NS & NS & NS & NS & * & NS & NS & NS & NS \\
\hline Q & & & NS & $*$ & NS & NS & $*$ & NS & NS & NS & NS & NS & NS \\
\hline
\end{tabular}

${ }^{2}$ Full bloom occurred 22 Apr. 1995. Treatments were applied 12 May when fruit was $8.4 \pm 0.60 \mathrm{~mm}$ in diameter. Tree spacing was $6.1 \mathrm{~m}$ between rows, $3.05 \mathrm{~m}$ tree width, and $4.3 \mathrm{~m}$ tree height, which was equivalent to $50 \% \mathrm{TRV}$.

y Two longest top shoots.

${ }^{x}$ Five longest scaffold shoots.

wShoots $>30 \mathrm{~cm}$ only.

"TCSA = trunk cross-sectional area.

Ns, $, * * *, * * *$ Nonsignificant or significant at $P \leq 0.05,0.01$, or 0.001 , respectively.

all 10 fruit on a measuring tape, and the $\mathrm{L}$ : D ratio was calculated. Fruit russet was rated on the following scale : $0=$ no lenticel development; $1=$ small lenticel enlargement; $2=$ lenticels rough and raised to touch; $3=$ some russet between lenticels; $4=$ considerable russetting other than lenticels; and $5=$ severe russet. Color was visually estimated as percentage of the surface showing red.

Expt. 6. In 1996, fifty-five 5-year-old 'Gala'/M. 27 trees (5 blocks) were used for 11 treatments. Spray thinning treatments (Table 6) were applied with or without prohexadione-calcium with a low-pressure hand wand sprayer 10,15 , and 20 DAFB. As described for Expt. 5, crop load was recorded and ten fruit were harvested from each tree on 27 Aug. and evaluated.

Expt. 7. Thirty 6-year-old 'Redhaven' peach trees (six blocks) were used for five treatments. Prohexadione-calcium $\left(375 \mathrm{mg} \cdot \mathrm{L}^{-1}\right)$ was applied : 1) alone; 2) with $0.125 \%$ Silwet L-77; 3) with $0.5 \% 70^{\circ}$ s oil; and 4 ) with $0.125 \% \mathrm{~L}-77+0.5 \% 70^{\circ} \mathrm{s}$ oil. Additional trees were left as controls. On 28 July, a sample of 10 fruit/tree were taken to determine fruit diameter, color, firmness, soluble solids, and weight, and length of the five longest shoots in the periphery of the tree were measured.

\section{RESULTS}

Expt. 1. In 1994, airblast applications of prohexadione-calcium reduced shoot length of 'Stayman'/seedling apple trees during the growing season. In the dormant season (7 Dec. 1994), all parameters of shoot growth measured were reduced (Table 1), except the diameter of the most terminal part of shoot (data not shown). Pruning time, weight, and number of cuts appeared to be reduced by treatment (data not shown), but differences were nonsignificant at $P \leq 0.05$. The trees were rather variable in crop load and quite vigorous, which might account for lack of significance. The fruit L : D ratio, flesh firmness, soluble solids, starch, red color, and fruit cracking were not affected by prohexadione-calcium.

Expt. 2. In 1995, prohexadione-calcium again reduced shoot length 
Table 3. Effects of prohexadione-calcium applied in 1996 on dormant season measurements and pruning of 'Starkrimson'/MM.106 and 'Starkrimson'/MM.111 trees in Jan. 1997.

\begin{tabular}{|c|c|c|c|c|c|c|c|c|c|c|c|c|}
\hline \multirow{4}{*}{$\begin{array}{l}\text { Prohexadione- } \\
\text { calcium } \\
\left(\mathrm{mg} \cdot \mathrm{L}^{-1}\right)\end{array}$} & \multirow{4}{*}{$\begin{array}{l}\text { Application } \\
\text { date }\end{array}$} & & & & & & \multirow{4}{*}{$\begin{array}{l}\text { Basal } \\
\text { diam } \\
(\mathrm{cm})\end{array}$} & & & \multicolumn{3}{|c|}{ Pruning } \\
\hline & & & & & & & & \multicolumn{2}{|c|}{ Nodes $/ \mathrm{m}$} & \multirow{3}{*}{$\begin{array}{c}\text { Time } \\
\left(\mathrm{min} / \mathrm{cm}^{2}\right. \\
\left.\text { TCSA }^{\mathrm{v}}\right)\end{array}$} & \multirow{3}{*}{$\begin{array}{c}\text { Wt/tree } \\
(\mathrm{kg})\end{array}$} & \multirow{3}{*}{$\begin{array}{l}\text { Cuts } \\
\text { tree }\end{array}$} \\
\hline & & \multicolumn{3}{|c|}{ Shoot length $(\mathrm{cm})$} & \multicolumn{2}{|c|}{ Shoot wt (g) } & & Basal 40 & Upper 30 & & & \\
\hline & & Top $^{y}$ & Scaffold $^{x}$ & $>30 \mathrm{~cm}^{\mathrm{w}}$ & Top $^{y}$ & Scaffold $^{x}$ & & $\mathrm{~cm}$ & $\mathrm{~cm}$ & & & \\
\hline 0 & & 118 & 115 & 985 & 148 & 304 & 12.2 & 54 & 84 & 6.0 & 2.52 & 106 \\
\hline 250 & 6 May & 65 & 74 & 462 & 53 & 145 & 10.2 & 86 & 128 & 3.3 & 0.84 & 55 \\
\hline 250 & 17 May & 96 & 85 & 627 & 91 & 155 & 9.8 & 75 & 97 & 5.2 & 1.52 & 83 \\
\hline 250,250 & 17 May, 3 June & 98 & 83 & 633 & 110 & 174 & 10.4 & 85 & 108 & 4.8 & 1.55 & 81 \\
\hline $250,250,250$ & 17 May, 3 June, 3 July & 75 & 70 & 555 & 72 & 160 & 11.0 & 95 & 112 & 4.3 & 1.32 & 78 \\
\hline $\begin{array}{l}250,250,250, \\
\quad 250\end{array}$ & $\begin{array}{l}17 \text { May, } 3 \text { June, } \\
3 \text { July, } 24 \text { July }\end{array}$ & 48 & 46 & 265 & 36 & 86 & 9.2 & 110 & 78 & 2.4 & 0.50 & 39 \\
\hline \multicolumn{13}{|l|}{ Contrasts } \\
\hline \multicolumn{2}{|c|}{0 vs. May 6250} & $* * *$ & $* * *$ & $* * *$ & $* * *$ & $* * *$ & $* * *$ & $* * *$ & NS & $* *$ & $* * *$ & $* *$ \\
\hline \multicolumn{2}{|c|}{0 vs. $250,250,250,250$} & $* * *$ & $* * *$ & $* * *$ & $* * *$ & $* * *$ & $* * *$ & $* * *$ & $* * *$ & $* * *$ & $* * *$ & $* * *$ \\
\hline \multicolumn{2}{|c|}{2506 May vs. 25017 May } & $* *$ & NS & $*$ & NS & NS & NS & $*$ & NS & $*$ & $*$ & NS \\
\hline \multicolumn{2}{|c|}{2506 May vs. 250,250} & $* *$ & NS & NS & $*$ & NS & NS & NS & NS & NS & NS & NS \\
\hline \multicolumn{2}{|c|}{2506 May vs. $250,250,250$} & NS & NS & NS & NS & NS & NS & $*$ & NS & NS & NS & NS \\
\hline \multicolumn{2}{|c|}{2506 May vs. $250,250,250,250$} & NS & NS & $* * *$ & NS & $*$ & $*$ & $* * *$ & $* * *$ & NS & NS & NS \\
\hline \multicolumn{13}{|l|}{ Regression } \\
\hline $\mathrm{L}$ & & $* * *$ & $* * *$ & $* * *$ & $* * *$ & $* * *$ & $* * *$ & $* * *$ & $* * *$ & NS & $* * *$ & $* * *$ \\
\hline $\mathrm{Q}$ & & $* * *$ & $* * *$ & $* * *$ & $* * *$ & $* * *$ & $* * *$ & $* * *$ & $* * *$ & NS & $* *$ & $* * *$ \\
\hline
\end{tabular}

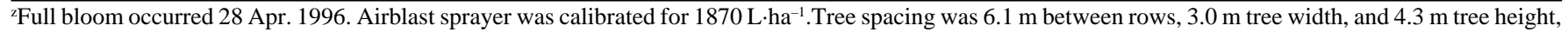
which was equivalent to $50 \%$ TRV.

yTwo longest top shoots.

${ }^{x}$ Five longest scaffold shoots.

${ }^{\text {w }}$ Shoots $>30 \mathrm{~cm}$ only.

vTCSA = trunk cross-sectional area.

ns, *,**, **** Nonsignificant or significant at $P \leq 0.05,0.01$, or 0.001 , respectively.

Table 4. Effects of prohexadione-calcium applied in 1997 on dormant season measurements and pruning of 'Ace Delicious'/MM111 trees in Dec. $1997 .{ }^{2}$

\begin{tabular}{|c|c|c|c|c|c|c|c|c|c|c|c|c|}
\hline \multirow{4}{*}{$\begin{array}{l}\text { Prohexadione- } \\
\text { calcium } \\
\left(\mathrm{mg} \cdot \mathrm{L}^{-1}\right)\end{array}$} & \multirow{4}{*}{$\begin{array}{c}\text { Application } \\
\text { date }\end{array}$} & & & & & & \multirow{4}{*}{$\begin{array}{l}\text { Basal } \\
\text { diam } \\
(\mathrm{cm})\end{array}$} & \multicolumn{5}{|c|}{ Pruning } \\
\hline & & \multirow{2}{*}{\multicolumn{3}{|c|}{ Shoot length $(\mathrm{cm})$}} & \multirow{2}{*}{\multicolumn{2}{|c|}{ Wt $(\mathrm{g})$}} & & \multicolumn{2}{|c|}{ Nodes/m } & \multirow{3}{*}{$\begin{array}{c}\text { Time/ } \\
\text { tree } \\
(\mathrm{min})\end{array}$} & \multirow{3}{*}{$\begin{array}{c}\mathrm{Wt} / \text { tree } \\
(\mathrm{kg})\end{array}$} & \multirow{3}{*}{$\begin{array}{c}\text { Cuts/ } \\
\text { tree }\end{array}$} \\
\hline & & & & & & & & \multirow{2}{*}{$\begin{array}{c}\text { Basal } 40 \\
\mathrm{~cm}\end{array}$} & \multirow{2}{*}{$\begin{array}{c}\text { Upper } 30 \\
\mathrm{~cm}\end{array}$} & & & \\
\hline & & Top $^{y}$ & Scaffold $^{x}$ & $>30 \mathrm{~cm}^{\mathrm{w}}$ & Top $^{y}$ & Scaffold $^{\mathrm{x}}$ & & & & & & \\
\hline $63,63,125$ & 25 Aprl., 2 May, 9 May & 71.2 & 65.0 & 1826 & 70.8 & 202 & 1.29 & 61 & 84 & 3.55 & 2.08 & 78.9 \\
\hline 125,125 & 25 Apr., 2 May & 58.8 & 61.2 & 1280 & 63.8 & 175 & 1.16 & 65 & 84 & 2.28 & 1.38 & 52.0 \\
\hline 250 & 25 Apr. & 64.4 & 71.0 & 1644 & 66.3 & 227 & 1.35 & 59 & 85 & 3.30 & 1.97 & 78.6 \\
\hline \multicolumn{13}{|l|}{ Comparisons } \\
\hline \multicolumn{2}{|c|}{0 vs. $63,63,125$} & NS & NS & NS & NS & NS & NS & NS & NS & NS & NS & NS \\
\hline \multicolumn{2}{|c|}{0 vs. 125,125 , } & NS & NS & $* *$ & NS & NS & $* *$ & $*$ & NS & $* * *$ & $* *$ & $* * *$ \\
\hline \multicolumn{2}{|l|}{0 vs. 250} & NS & NS & $*$ & NS & NS & NS & NS & NS & $*$ & NS & NS \\
\hline \multicolumn{2}{|l|}{0 vs. 125} & NS & NS & $*$ & NS & NS & NS & NS & NS & $*$ & NS & NS \\
\hline \multicolumn{2}{|c|}{$63,63,125$ vs. 125,125} & NS & NS & NS & NS & NS & * & NS & NS & $*$ & NS & NS \\
\hline \multicolumn{2}{|c|}{$63,63,125$ vs. 250} & NS & NS & NS & NS & NS & NS & NS & NS & NS & NS & NS \\
\hline
\end{tabular}

${ }^{2}$ Full bloom occurred 15 Apr. 1997. Data taken on treatments Dec. 1997.

${ }^{\mathrm{y}}$ Airblast sprayer was calibrated for $1870 \mathrm{~L} \cdot \mathrm{ha}^{-1}$. Tree spacing was $6.1 \mathrm{~m}$ between rows, $3.0-\mathrm{m}$ tree width and $4.3-\mathrm{m}$ tree height, which was equivalent to $50 \% \mathrm{TRV}$. Ns, ${ }^{* * *, * * *}$ Nonsignificant or significant at $P \leq 0.05,0.01$, or 0.001 , respectively.

(Table 2). The most effective treatments, $500 \mathrm{mg} \cdot \mathrm{L}^{-1}$ at either 1870 or $468 \mathrm{~L} \cdot \mathrm{ha}^{-1}$, reduced pruning weight and pruning time, but not number of cuts. The trees were again rather variable in crop load and tree vigor which may have accounted for the lack of significance in some cases. Fruit L : D ratio, soluble solids, and starch content were not affected by prohexadione-calcium, but fruit firmness and red color were slightly increased (data not shown). Neither weight per fruit nor yield per tree was affected by the chemical (data not shown).

Expt. 3. In 1996, multiple applications generally controlled tree growth better than did a single application (Table 3). Regrowth of the strongest shoots was observed as new light green leaves developing on the most vigorous shoots just before the second, third, or fourth applications. No differences were found in fruit diameter, red color, number of fruit per tree, fruit weight, or crop load (data not shown).

Expt. 4. In 1997, rates for multiple applications were greatly reduced from the 1996 rates, but were applied sooner after bloom. Growth and crop load were rather variable among trees, which resulted in fewer statistical differences than expected (Table 4). The crop load on these trees was very light because of a prebloom freeze; this further increased variability. For this reason fruit quality measurements were not taken. Control trees required more time to prune than did prohexadione-calcium-treated trees. Two applications at $125 \mathrm{mg} \cdot \mathrm{L}^{-1}$ gave better growth control than did three treatments of 63, 63, and 125 $\mathrm{mg} \cdot \mathrm{L}^{-1}$. These data further reinforce the need for adequate amounts of chemical, and possibly multiple applications, being applied soon after bloom (see also Expt. 3, Table 3) to achieve the optimum vegetative growth control.

Expt. 5. In 1995, prohexadione-calcium significantly increased thinning response to ethephon (treatments 8 vs. 9), but not to the combinations of carbaryl + Accel $^{\circledR}$ (treatments 2,4 vs. 5, 7) or Vydate + Accel $^{\circledR}$, (treatments 3 vs. 6) (Table 5). No differences in fruit diameter, L : D ratio, russet, or color were found (data not shown).

Expt. 6. In 1996, NAA thinned significantly at 10 and 15 DAFB, but not at $20 \mathrm{DAFB}$, but neither Accel $^{\circledR}$, nor carbaryl was effective. 
Table 5. Effect of prohexadione-calcium on response of 'Fuji'/M.27 to chemical thinning agents (1995). ${ }^{2}$

\begin{tabular}{|c|c|c|c|c|c|}
\hline \multirow[b]{2}{*}{ Treatment } & \multirow[b]{2}{*}{$\begin{array}{l}\text { Thinning } \\
\text { agent }^{\mathrm{y}}\end{array}$} & \multicolumn{3}{|c|}{ Added chemicals } & \multirow{2}{*}{$\begin{array}{c}\text { Fruit } / \mathrm{cm}^{2} \text { limb } \\
\text { cross-sectional } \\
\text { area (30 May) }\end{array}$} \\
\hline & & $\begin{array}{c}\text { Prohexadione- } \\
\text { calcium }\end{array}$ & Silwet & Oil & \\
\hline$\overline{1 .}$ & Control & - & - & - & 8.30 \\
\hline 2. & Carbaryl + Accel & - & - & + & 0.34 \\
\hline 3. & Carbaryl + Accel & + & - & + & 1.08 \\
\hline 4. & Carbaryl + Accel & - & + & - & 3.10 \\
\hline 5. & Carbaryl + Accel & + & + & - & 2.47 \\
\hline 6. & Vydate + Accel & - & - & - & 2.71 \\
\hline 7. & Vydate + Accel & + & - & - & 1.76 \\
\hline 8. & Ethephon & - & - & - & 4.87 \\
\hline 9. & Ethephon & + & - & - & 1.99 \\
\hline 10. & Hand thinned & - & - & - & 4.74 \\
\hline \multicolumn{6}{|c|}{ Contrasts: prohexadione-calcium vs. none } \\
\hline 2 vs. 3 & & & & & NS \\
\hline 4 vs. 5 & & & & & NS \\
\hline 6 vs. 7 & & & & & NS \\
\hline 8 vs. 9 & & & & & $* *$ \\
\hline $2,4,6$ vs. 3 & 7 & & & & NS \\
\hline $2,4,6,8 \mathrm{vs}$ & $, 5,7,9$ & & & & $*$ \\
\hline
\end{tabular}

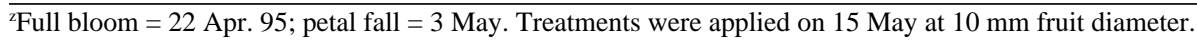
${ }^{y}$ Concentrations $\left(\mathrm{mg} \cdot \mathrm{L}^{-1}\right)$ : carbaryl-1800; Accel-50; Vydate-300; ethephon-500.

Ns, *,****** Nonsignificant or significant at $P<0.05,0.01$, or 0.001 , respectively.

Table 6. Effects of prohexadione-calcium on response of 'Gala'/M.27 to chemical thinning agents (1996).

\begin{tabular}{|c|c|c|c|c|c|}
\hline Treatment $^{\mathrm{y}}$ & $\begin{array}{c}\text { Thinning } \\
\text { agent }\end{array}$ & $\begin{array}{l}\text { Prohexadione- } \\
\text { calcium }^{\mathrm{y}}\end{array}$ & $\begin{array}{c}\text { Spray } \\
\text { timing } \\
(\mathrm{DAFB})^{\mathrm{z}}\end{array}$ & $\begin{array}{c}\text { Fruit/cm }{ }^{2} \text { limb } \\
\text { cross-sectional } \\
\text { area ( } 7 \text { June) }\end{array}$ & $\begin{array}{c}\text { Fruit } \\
\text { diam }(\mathrm{cm}) \\
(8 \mathrm{Aug}) \\
\end{array}$ \\
\hline 1. & Control & & & 12.8 & 6.94 \\
\hline 2. & NAA & - & +10 & 8.7 & 7.23 \\
\hline 3. & NAA & + & +10 & 3.9 & 7.12 \\
\hline 4. & NAA & - & +15 & 6.6 & 7.21 \\
\hline 5. & NAA & + & +15 & 8.2 & 7.26 \\
\hline 6. & NAA & - & +20 & 11.4 & 6.85 \\
\hline 7. & NAA & + & +20 & 11.9 & 6.98 \\
\hline 8. & Carbaryl & - & +20 & 10.0 & 6.67 \\
\hline 9. & Carbaryl & + & +20 & 14.3 & 6.95 \\
\hline 10. & Accel & - & +20 & 12.8 & 6.94 \\
\hline 11. & Accel & + & +20 & 14.9 & 6.79 \\
\hline Contrasts: & \multicolumn{5}{|c|}{$\underline{\text { Comparisons }}$} \\
\hline 2,3 vs. 4,5 & \multicolumn{3}{|c|}{10 vs. 15 (DAFB) } & NS & NS \\
\hline 2,3 vs. 6,7 & \multicolumn{3}{|c|}{10 vs. 20 (DAFB) } & NS & NS \\
\hline 4,5 vs. 6,7 & \multicolumn{3}{|c|}{15 vs. 20 (DAFB) } & $* * *$ & $* *$ \\
\hline 6,7 vs. 8,9 & \multicolumn{3}{|c|}{ NAA vs. carbaryl } & NS & NS \\
\hline 6,7 vs. 10,11 & \multicolumn{3}{|c|}{ NAA vs. Accel } & $\mathrm{NS}$ & NS \\
\hline 8,9 vs. 10,11 & \multicolumn{3}{|c|}{ Carbaryl vs. Accel } & NS & NS \\
\hline 2 vs. 3 & \multicolumn{3}{|c|}{$\begin{array}{l}\text { Prohexadione-calcium vs. none } \\
\text { (10 DAFB) }\end{array}$} & NS & NS \\
\hline 4 vs. 5 & \multicolumn{3}{|c|}{ (15 DAFB) } & NS & NS \\
\hline 6 vs. 7 & \multicolumn{3}{|c|}{ (20 DAFB) } & NS & NS \\
\hline $2,4,6$ vs. $3,5,7$ & \multicolumn{3}{|c|}{$\begin{array}{l}\text { NAA vs NAA+ prohexadione- } \\
\text { calcium }(10,15,20, \text { DAFB })\end{array}$} & NS & NS \\
\hline $\begin{array}{l}2,4,6,8,10 \mathrm{vs} \\
\quad 3,5,7,9,11\end{array}$ & \multicolumn{3}{|c|}{ All thinners vs. all thinners } & NS & NS \\
\hline
\end{tabular}

${ }^{2}$ Full bloom occurred 30 Apr. 1996; DAFB = days after full bloom.

${ }^{y}$ Concentrations $\left(\mathrm{mg} \cdot \mathrm{L}^{-1}\right)$ used: NAA-15: carbaryl-600; Accel-50 ; prohexadione-calcium-250.

Ns, ${ }^{*}, * *, * * *$ Nonsignificant or significant at $P<0.05,0.01$, or 0.001 , respectively.

Prohexadione-calcium had no effect on fruit set in response to NAA, Accel $^{\circledR}$, or carbaryl (Table 6), or on fruit characteristics or subsequent season flowering.

Expt. 7. One application of prohexadione-calcium applied 2 May on 'Redhaven' peach trees did not affect shoot length of the five longest shoots on the tree, or fruit diameter, weight, color, firmness, or soluble solids (data not shown). Adjuvants L-77, oil, or oil + L-77 did not affect the efficacy of the chemical (data not shown).

\section{DISCUSSION}

Since $\approx 75 \%$ of apple tree shoot growth occurs in the first 30 days after bloom (Expt. 1), maximum suppression of tree growth can only be achieved if the first flush of growth is controlled. Other growth retardants also suppress shoot growth of apple trees more when applied early in the growing season (Miller, 1988). Adequate prohexadione-calcium $\left(125 \mathrm{mg} \cdot \mathrm{L}^{-1}\right)$ was required in the first and subsequent applications to maximize shoot growth control (Expt. 3). Prohexadione-calcium suppression of shoot growth was short lived, and shoots with the greatest vigor began growing again within a few of weeks of application (Expts. 3 and 4). Trees in highest vigor (due to a partial crop load, vigorous rootstocks, good soil moisture, etc.) may require weekly monitoring to determine the number and timing of additional applications to prevent regrowth. Even though the most vigorous shoots on the trees were used for most of the shoot growth measurements reported in this paper (Expts. 1-4), the number of cuts required to prune the tree may be more reflective of the degree of or shoot growth suppression, since less vigorous shoot are less likely to regrow. Prohexadione-calcium did not promote enlargement of terminal buds or basal shoot diameter as did daminozide [butanedioic acid 
mono(2,2-dimethylhydrazide)](Emerson, unpublished). The enlarged terminal buds and shoot diameters of daminozide-treated trees may explain why daminozide sprays did not control shoot growth in subsequent seasons. Additional studies are needed to determine if any subsequent season carry-over effects are noticed in prohexadionecalcium-treated trees; the reduced terminal bud size and shoot diameters of such trees suggest a reduction in tree vigor.

Very little if any direct effect on fruit firmness, soluble solids, starch content, shape (Expts. 1-4), or fruit cracking (Expt. 1) was observed with the rates of prohexadione-calcium used in these studies. The small increase in red color probably resulted from better light penetration because of vegetative growth suppression rather than being a direct effect on fruit coloring. Thinning activity of NAA, carbaryl, or Accel was not affected by tank mixing prohexadionecalcium with chemical thinning sprays applied to 'Gala'/M.27 trees 20 DAFB (Expt. 6). Tank mixing prohexadione-calcium with combinations of NAA, Vydate + Accel, or carbaryl + Accel + oil also did not alter fruit thinning of 'Fuji'/M.27 when applied at 10-mm fruit diameter (Expt. 5). In one experiment, prohexadione-calcium potentiated thinning by ethephon when applied to 'Fuji'/M.27 (Expt. 5). Interference of prohexadione-calcium with chemical thinning or natural fruit set was found to be minimal, if any, in these experiments (Expts. 5 and 6). Several years of commercial experience will be required to determine if this growth regulator can eliminate or reduce the need to annually prune apple trees, while maintaining fruit quality and production.

\section{Literature Cited}

Faust, M. (ed.). 1984 International workshop on controlling vigor in fruit trees. Acta Hort. 146.

Looney, N.E. 1983. Growth regulator usage in apple and pear production, p. 27-40 In: L.G. Nickell (ed.). Plant growth regulating chemicals. vol. I. CRC Press, Boca Raton, Fla.

Luckwill, L.C. 1970. The control of growth and fruitfulness of apple trees. p. 237-254. In: L.C. Luckwill and C.V. Cutting (eds.). Physiology of tree crops. Academic, London.

Miller, S. S. 1988. Plant bioregulators in apple and pear culture. Hort. Rev. 10:309-401.

Poapst, P.A., G.M. Ward, and W.R. Phillips. 1959. Maturation of McIntosh apples in relation to starch loss and abscission. Can. J. Plant Sci. 39:257263.

SAS Institute. 1985. SAS user's guide: Statistics. SAS Inst., Cary, N.C.

Williams, M.W. 1984. Use of bioregulators to control vegetative growth of fruit trees and improve fruit efficiency, p. 93-99. In: R.L. Orydn and F.R. Rittig (eds.). Bioregulators: Chemistry and uses. Amer. Chem. Soc. Washington, D.C. 